'Departamento de Radiología, Clínica Las Condes. Santiago, Chile. ${ }^{2}$ Departamento de Gastroenterología, Hospital San Juan de Dios. Santiago, Chile. 3Departamento de Cirugía, Clínica Las Condes. Santiago, Chile.

Trabajo no recibió financiamiento. Los autores declaran no tener conflictos de interés.

Recibido el 9 de marzo de 2019, aceptado el 19 de julio de 2019.

Correspondencia a: Dr. David Ladrón de Guevara H. Servicio de Radiología, Clínica Las Condes. Santiago, Chile. dlg@clc.cl

\section{Cáncer sincrónico detectado con PET/ CT-Tomografía Computada contrastada (PET/CTc) en 210 pacientes con cáncer colorrectal recientemente diagnosticado}

\author{
DAVID LADRÓN DE GUEVARA ${ }^{1}$, DANIEL PÉREZ ${ }^{1}$, PAULINA NÚÑEZ ${ }^{2}$, \\ FELIPE RAMÍREZ ${ }^{1}$, ALEJANDRO ZÁRATE ${ }^{3}$, FRANCISCO LÓPEZ ${ }^{3}$
}

\begin{abstract}
Background: Colorectal cancer (CRC) is the third most prevalent cancer in the world and is the second cause of cancer death. Positron emission tomography/computed tomography (PET/CT) using ${ }^{18} F-F D G$ is used for its staging and follow up. Aim: To assess the occurrence of synchronous colonic and extracolonic tumors detected with contrast-enhanced F18-FDG PET/CT (PET/CTc) in patients with a recently diagnosed CRC. Material and Methods: PET/CTc of 210 patients aged 16-91 years (63\% men) with a recently diagnosed CRC were reviewed. PET/CTC with incidental findings, not expected to be due to CRC, were followed (laboratory, imaging and pathology) searching for synchronous tumors. Results: Ten patients (4,7\%) had a second synchronous CRC. Only 70\% of synchronous CRC were accessible to colonoscopy, due mainly to incomplete procedures for stenotic tumors. Extracolonic synchronous neoplasms were detected in 12 patients (5,7\%), namely lung cancer in three, renal cell carcinoma in two, non-Hodgkin lymphoma in two, pancreatic cancer in one, breast cancer in one, hepatocellular carcinoma in one, bladder cancer in one and thyroid cancer in one. Conclusions: Ten percent of patients with a recently diagnosed CRC had a synchronic neoplasm detected at staging using PET/CTc.
\end{abstract}

(Rev Med Chile 2019; 147: 828-835)

Key words: Colonic Neoplasms; Colorectal Neoplasms; Neoplasms, Multiple Primary.
E 1 cáncer colorrectal (CCR) es la tercera neoplasia más frecuente y representa la segunda causa de muerte por cáncer en el mundo ${ }^{1}$. En Chile, las neoplasias digestivas son responsables de $41,8 \%$ de las muertes por neoplasias, siendo el CCR la segunda en frecuencia luego del cáncer gástrico. La mortalidad por cáncer de colon ha aumentado en la última década de 5,9 a 6,7 por 100 mil habitantes en nuestro país, al igual que el cáncer de recto (de 1,7 a 1,9 por 100 mil habitantes) ${ }^{2}$. La incidencia del CCR es levemente mayor en hombres, con una proporción de 1,4:12-4.

Se estima que alrededor de $20-25 \%$ de los pacientes con CCR presenta metástasis hematógenas al momento del diagnóstico, más frecuentemente a nivel hepático y pulmonar ${ }^{5-7}$. La presencia de metástasis a distancia limita significativamente el número de pacientes que podrán optar a trata- 
miento quirúrgico curativo, y se asocia a menor sobrevida ${ }^{3,5,6}$.

Otro factor importante que puede modificar el tratamiento es la presencia de tumores malignos o premalignos colorrectales sincrónicos, hecho que determinaría un cambio en la conducta quirúrgica $^{8}$. La incidencia de tumores sincrónicos colorrectales varía entre 1,3 y 7,3\% $\%^{8-11}$ según literatura internacional. En una casuística latinoamericana de 86 pacientes con CCR, Ruffinengo et $\mathrm{a}^{12}$ describe que 5,8\% de ellos presentó un tumor sincrónico pesquisado mediante colonoscopia. No encontramos literatura nacional al respecto.

Un área muy poco explorada en pacientes con CCR es la existencia de neoplasias sincrónicas extracolónicas. Su incidencia ha sido descrita en escasas publicaciones, y varía entre $0,9 \%$ a $4,5 \%^{13-15}$, aunque las metodologías de búsqueda fueron diferentes, no estandarizadas o no descritas entre las diferentes series, y la mayoría antes del desarrollo de técnicas híbridas como el PET/ CT. Se ha reportado una incidencia de neoplasias sincrónicas extracolónicas en $0,35 \%$ de los pacientes asintomáticos sometidos a tamizaje mediante colonoscopía virtual ${ }^{16}$.

La etapificación imagenológica de los pacientes con CCR se realiza habitualmente con tomografía computada (TC) de tórax, abdomen y pelvis, y en algunos casos con resonancia magnética (RM). El PET/CT no figura como examen de primera línea en el estudio inicial de CCR ${ }^{17,18}$.

Si bien se ha demostrado la mayor sensibilidad del PET/CT en detección de metástasis extrahepáticas respecto a la etapificación tradicional ${ }^{19}, \mathrm{y}$ su significativo impacto en el manejo de pacientes oligometastásicos, encontrando siembra secundaria extrahepática en $25 \%$ de los pacientes con aparente metástasis única hepática ${ }^{18,20}$, las guías aún no apoyan su uso extendido en etapificación de CCR, restringiéndose sólo a algunas situaciones, como pacientes oligometastásicos con opción de cirugía curativa que incluya metastasectomía ${ }^{17}$, o en lesiones hepáticas o extrahepáticas dudosas con la TC y/o RM ${ }^{18}$. Las desventajas del PET/CT que se mencionan en la literatura son su baja especificidad y la ocurrencia de falsos positivos ${ }^{20}$, ya que procesos inflamatorio-infecciosos, lesiones benignas y aún situaciones fisiológicas pueden ocasionar hipercaptación del radiotrazador ${ }^{20}$.

La posibilidad de realizar ambos estudios, PET/ CT y TC contrastada (PET/CTc), de una sólo vez en el mismo equipo y sin mover al paciente, y de interpretar multidisciplinariamente (radiólogo y médico nuclear) ambas imágenes en su conjunto, crea un nuevo paradigma imagenológico que podría aumentar la detección de lesiones y al mismo tiempo mejorar la especificidad, dado la complementariedad de ambas técnicas. Esto pudiera ser válido no sólo en la detección de metástasis, sino también en la pesquisa de neoplasias sincrónicas.

El objetivo de este estudio es determinar la frecuencia de tumores malignos sincrónicos colorrectales y extracolónicos en población con CCR que se realizó un PET/CTc de etapificación.

\section{Pacientes y Método}

Se revisó la base de datos de PET/CT de nuestra institución, seleccionando los estudios PET/CTc corporales realizados con flúor 18 -fluorodeoxiglucosa (F18-FDG) entre diciembre de 2008 y diciembre de 2017, que fueran derivados con diagnóstico de cáncer de colon o recto en etapificación. Sólo se incluyeron aquellos pacientes con confirmación histológica de carcinoma de colon o recto. Para los efectos de este trabajo se consideraron sólo los pacientes con adenocarcinoma, excluyendo a aquellos con otras histologías. Los pacientes con antecedentes de poliposis familiar o síndromes hereditarios fueron excluidos.

Aquellos PET/CTc que mostraron hallazgos incidentales, no atribuibles al tumor primario colorrectal ni a sus aparentes metástasis, fueron investigados dirigidamente en busca de confirmación o exclusión del incidentaloma visible al PET/CTc. Se revisó la ficha electrónica en busca de controles clínicos post-tratamiento, exámenes de laboratorio (antígeno prostático específico, CEA, CA19-9), procedimientos invasivos (toracotomías, mediastinoscopias, endoscopias, biopsias o citologías), exámenes de imagen de seguimiento (ecografía, TC, RM, PET/CT) e histopatología de lodos los pacientes.

\section{Adquisición del PET/CT-tomografía computada contrastada (PET/CTc)}

Los PET/CTc fueron realizados en un equipo GE Discovery STE de 16 canales, con adquisición de imágenes desde el vértex craneano hasta rodillas. Se inyectó $0,1 \mathrm{mCi} / \mathrm{kg}$ de $\mathrm{F} 18-\mathrm{FDG}$, previo ayuno de $6 \mathrm{~h}$ y suspensión de metformina $48 \mathrm{~h}$ antes del examen. Inmediatamente después de la 
adquisición del PET/CT y sin mover al paciente, se realizó una TC contrastada de cerebro, cuello, tórax, abdomen y pelvis, que incluyó fase arterial y porto-venosa para el abdomen, en todos los pacientes. Las imágenes fueron interpretadas por un especialista doble radiólogo y médico nuclear, con apoyo en radiólogos subespecialistas (radiólogo de tórax y neuro-radiólogo) según el caso.

\section{Definición de cáncer sincrónico}

Un cáncer sincrónico colorrectal se definió según los criterios de Warren y Gates ${ }^{10,21}$, como: 1) adenocarcinoma probado histológicamente; 2) claramente separado espacialmente del CCR original; 3) donde se haya excluido que corresponda a una metástasis de éste. El tumor sincrónico debe ser contemporáneo al CCR original o ser pesquisado con menos de 6 meses de diferencia ${ }^{10,21}$.

Se consideró tumor sincrónico extracolónico aquel cáncer de pesquisa incidental, confirmado histológicamente como distinto al cáncer de colon, en cualquier ubicación fuera del colon y recto, visible en cualquiera de las fases del examen híbrido, tanto en las imágenes de PET/CT como en la TC contrastada. Se excluyeron los casos con neoplasias extracolónicas conocidas, que estuvieran en control o en tratamiento previo al PET/CTc.

\section{Análisis estadístico}

Ya que se trata de un estudio descriptivo, no se utilizó estadística analítica. Los hallazgos incidentales del PET/CTc se clasificaron en: 1) verdaderos positivos: hallazgos confirmados como neoplasias; 2) falsos positivos: confirmados como lesiones no neoplásicas, inflamatorias o fisiológicas; 3 ) no confirmados: no estudiados o con estudios no concluyentes.

\section{Aspectos éticos}

La selección de los pacientes se realizó utilizando la "base de datos PET/CT de CLC", aceptada por el Comité de Ética de Clínica Las Condes el $1^{\circ}$ de agosto de 2014. Todos los pacientes incluidos en la base de datos firmaron un consentimiento informado respecto a la utilización de sus imágenes de manera anónima para fines de investigación.

\section{Resultados}

La población con CCR que se efectuó etapificación mediante PET/CTc fue de 217, de los cuales 210 correspondieron a adenocarcinoma (mediana
65 años, rango 16-91 años, 63\% hombres), 4 a carcinoma escamoso rectal o anorrectal (mediana 61 años, rango 43-84 años, 50\% hombres), y 3 a carcinoma neuroendocrinos (mediana 69 años, rango 58-78 años, 67\% hombres). Estos dos últimos grupos, con histología diferente a adenocarcinoma, fueron excluidos del análisis.

Treinta y seis coma dos porciento de los pacientes (76/210) presentó metástasis hematógenas al momento del diagnóstico, las más frecuentes hepáticas $(25,7 \%)$, pulmonares $(14,8 \%)$ y peritoneales $(9,5 \%)$.

No todos los pacientes con CCR tuvieron una colonoscopía previa al PET/CTc. En 4 casos de presentación aguda del cuadro, ya sea por perforación u obstrucción intestinal, la colonoscopía preoperatoria fue omitida. El PET/CTc antecedió a la cirugía en todos los casos. Considerando los casos en que se tuvo acceso al informe competo de la colonoscopía preoperatoria (126 pacientes), $54 \%$ de ellos fue descrita como colonoscopía completa, y $46 \%$ incompleta.

De los pacientes con adenocarcinoma colorrectal, 4,7\% (10/210) presentaron un segundo tumor colorrectal sincrónico, todos ellos adenocarcinomas, ubicados en colon transverso (4), ciego (2), recto (2), sigmoides (1), y descendente (1). El 70\% de las lesiones sincrónicas se ubicaron en un segmento proximal del colon respecto al CCR conocido. Sólo 40\% de los tumores fueron visibles con la TC contrastada, y sólo $70 \%$ estuvo al alcance de la colonoscopia preoperatoria (Tabla 1). En un caso el PET/CT no detectó el tumor sincrónico debido a la alta captación intestinal difusa de F18FDG por no suspensión de metformina, aunque fue visible retrospectivamente en la TC, y visible en un segundo PET/CTc realizado con adecuada preparación un mes después.

El PET/CT detectó además 14 pólipos sincrónicos en 9 pacientes, que fueron confirmados como adenomas con displasia leve (3), moderada (5) o severa (6). Ninguno de ellos fue visible con TC contrastada, y sólo $57 \%$ pudo ser detectado con la colonoscopía preoperatoria, confirmándose posteriormente con resección quirúrgica, colonoscopía intraoperatoria o colonoscopía postoperatoria diferida.

Se encontró un cáncer sincrónico extracolónico en $5,7 \%$ de los pacientes con adenocarcinoma colorrectal $(12 / 210)$, representados por: cáncer pulmonar (3), carcinoma de células renales (2), 
Tabla 1. Tumores sincrónicos detectados con PET/CTc en pacientes con adenocarcinoma colorrectal y positividad según examen

\begin{tabular}{|c|c|c|c|c|}
\hline Tumores sincrónicos & n tumores & $\begin{array}{c}\text { Visibles con } \\
\text { PET }\end{array}$ & $\begin{array}{c}\text { Visibles con } \\
\text { TCC }\end{array}$ & $\begin{array}{l}\text { Visibles con } \\
\text { colonoscopia }\end{array}$ \\
\hline Sincrónicos colorrectales & $\mathrm{n}=10$ & $9(90 \%)$ & $4(50 \%)$ & $7(70 \%)$ \\
\hline - Ciego & 2 & 1 & 2 & 1 \\
\hline - C. Transverso & 4 & 4 & 0 & 3 \\
\hline - C. Descendente & 1 & 1 & 0 & 1 \\
\hline - C. Sigmoides & 1 & 1 & 1 & 0 \\
\hline - Recto & 2 & 2 & 2 & 2 \\
\hline Pólipos sincrónicos & $n=14$ & $14(100 \%)$ & $0(0 \%)$ & $8(57 \%)$ \\
\hline - Displasia leve & 3 & 3 & 0 & 2 \\
\hline - Displasia moderada & 5 & 5 & 0 & 3 \\
\hline - Displasia severa & 6 & 6 & 0 & 3 \\
\hline Sincrónicos extracolóncos & $\mathrm{n}=12$ & $8(67 \%)$ & $11(92 \%)$ & \\
\hline - Pulmón & 3 & 3 & 3 & \\
\hline - Renal & 2 & 0 & 2 & \\
\hline • LNH & 2 & 2 & 2 & \\
\hline - Mama & 1 & 1 & 1 & \\
\hline - Tiroides & 1 & 1 & 0 & \\
\hline - Páncreas & 1 & 1 & 1 & \\
\hline - $\mathrm{HCC} * *$ & 1 & 0 & 1 & \\
\hline - Vejiga & 1 & 0 & 1 & \\
\hline Sincrónicos de bajo grado & $\mathrm{n}=6$ & 0 & $6(100 \%)$ & \\
\hline - Meningioma* & 4 & 0 & 4 & \\
\hline - TNE páncreas** & 2 & 0 & 2 & \\
\hline
\end{tabular}

LNH: Linfoma no Hodgkin, HCC: Hepatocarcinoma, TNE: Tumor neuroendocrino. * Un paciente presentó dos meningiomas. **El HCC y los TNE de páncreas fueron visibles sólo en fase arterial de la TC.

linfoma no Hodgkin (2), adenocarcinoma de páncreas (1), cáncer de mama (1), hepatocarcinoma (1), carcinoma urotelial vesical (1), y cáncer tiroideo (1). En todos estos casos se realizó tratamiento diferido, quirúrgico y/o quimioterapia, de los tumores sincrónicos encontrados. La positividad del PET y TC contrastada para cada lesión se muestra en la Tabla 1.

En todos los casos de tumores sincrónicos, colónicos o extracolónicos, y de pólipos displásicos colorrectales, se realizó confirmación histológica y tratamiento quirúrgico o quimioterapia (en casos de linfoma) con intención curativa.

Excluyendo los pólipos displásicos colorrectales, $10,5 \%$ de los pacientes con adenocarcinoma colorrectal presentaron un tumor sincrónico al momento del diagnóstico.
Las lesiones tumorales de bajo grado detectadas con el PET/CTc no fueron incluidas en dicho porcentaje, tales como 4 meningiomas en 3 pacientes, y 2 tumores neuroendocrinos del páncreas en 2 pacientes.

Del total de hallazgos incidentales al PET/ CTc, 34\% (26 casos) no fue confirmado. Estos casos correspondieron mayormente a pacientes tratados en otros centros, y que sólo arribaron a nuestro establecimiento a realizarse el PET/CTc, sin posibilidad de seguimiento. En 9 casos (12\%) el PET/CTc arrojó falsos positivos, correspondientes a actividad fisiológica de colon, lesiones pulmonares inflamatorias/infecciosas, endometriosis pelviana y colecistitis crónica. Los resultados del total de incidentalomas del PET/CTc se muestra en la Figura 1. 


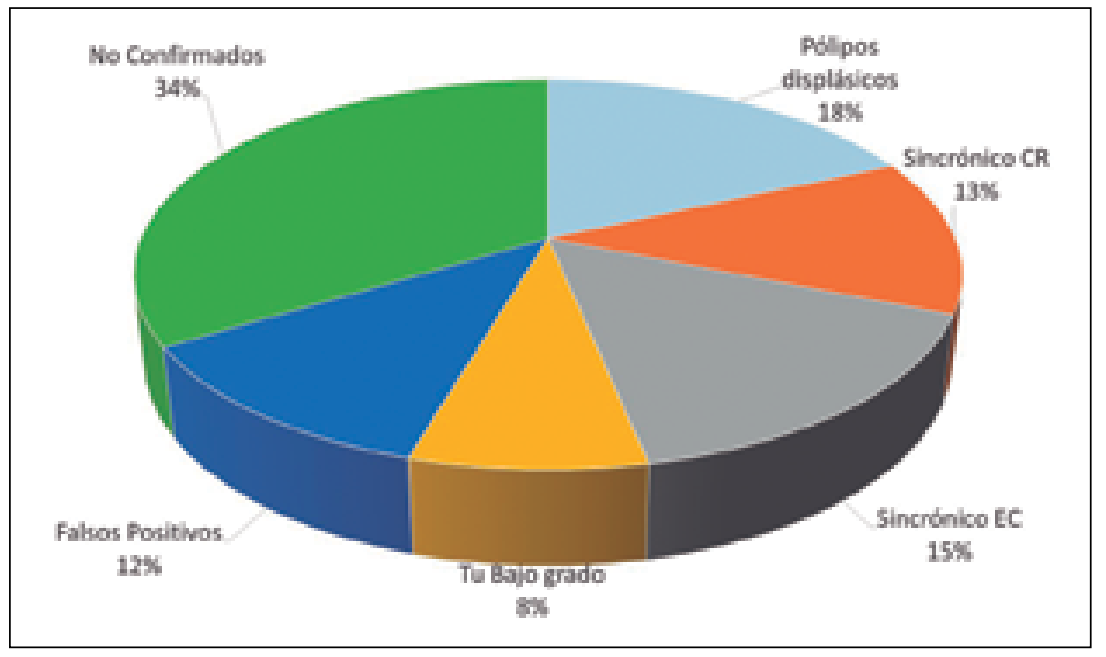

Figura 1. Distribución de frecuencia de los incidentalomas detectados con PET/CTc en pacientes con cáncer colorrectal en etapificación. CR: colorrectal; EC: Extracolónico.

\section{Discusión}

En nuestra casuística, el 10,5\% de los pacientes con CCR en etapificación presentó un cáncer sincrónico detectado con técnica híbrida PET/CTc. Estos correspondieron tanto a tumores malignos colorrectales, como a lesiones extracolónicas.

\section{Cáncer sincrónico colorrectal}

La coexistencia de varias lesiones malignas colorrectales sincrónicas está bien documentada en la literatura, y representa 1,3-7,3\% de los pacientes que se realizan estudio prequirúrgico por cáncer colorrectal ${ }^{8-10}$. Nuestra cifra de $4,7 \%$ es concordante con $4 \%$ reportado en artículos de revisión actuales ${ }^{18,22}$.

Las estadísticas de tumores sincrónicos son diferentes según como se defina el grupo a estudiar, si consideran o no tumores hereditarios y/o según el tipo de lesión incluida (adenoma/displasia/cáncer in situ o invasor). En un estudio poblacional danés ${ }^{11}$ se demostró 1,3\% de tumores sincrónicos en mayores de 50 años con cáncer no hereditario. Piñol et $\mathrm{al}^{9}$ en 1.522 pacientes, detectaron tumores sincrónicos en $33,2 \%$, aunque ellos consideraron todo tipo de lesiones, incluida las benignas (adenoma $=411$, carcinoma $=27 \mathrm{y}$ ambos $=67)$. Tomando en cuenta solo aquellas malignas $(27+67)$, se habría detectado $6,2 \%$ de tumores sincrónicos. Ellos describen que los factores de riesgo más relevantes son el género masculino $\mathrm{OR}=1,94 \mathrm{IC}$
95\% (1,43-2,65), historia personal de adenoma OR $=3,39$ IC 95\% $(1,58-7,31)$ y ubicación proximal OR $=1,4$ IC 95\% $(1,02-1,94)$.

En una serie de 384 pacientes con CCR, Borda et $\mathrm{a}^{23}$ describen que $7,3 \%$ presentó un cáncer colorrectal sincrónico, y $54,4 \%$, uno o más adenomas sincrónicos. Ellos destacan el hecho que $43 \%$ de las lesiones sincrónicas se ubicaron proximales a la lesión principal y una tercera parte de ellos eran tumores avanzados. Por esto recomiendan fuertemente realizar colonoscopía completa en todos los casos en que sea posible.

Se estima que hasta $10 \%$ de las colonoscopías de tamizaje realizadas a población sana son incompletas, es decir no alcanzan el ciego ${ }^{24}$. Por otro lado, 10 a $29 \%$ de los CCR son obstructivos ${ }^{25}$, impidiendo el paso del colonoscopio hacia los segmentos más proximales del colon. En estos casos, se recomienda utilizar estudios de imagen tales como colonografía con tomografía computada ${ }^{26}$, o también llamada "colonoscopia virtual", y PET/ $\mathrm{CT}$ con F18-FDG ${ }^{27}$. Otras alternativas como la colonoscopia intraoperatoria o simple palpación quirúrgica también son posibles de llevar a cabo ${ }^{26}$.

El PET/CT ha sido sugerido en la literatura como un nuevo método de pesquisa de lesiones sincrónicas proximales al sitio obstructivo ${ }^{28,29}$, pero falta mayor evidencia para establecer una recomendación formal. En un estudio retrospectivo japonés de 93 pacientes con CCR obstructivo se confirmaron por histología 83 lesiones proxima- 
les $^{27}$. El PET/CT mostró una sensibilidad de 25,3\% y un valor predictivo positivo de $77,8 \%$ para el total de las lesiones. Sin embargo, al desglosar según tamaño tumoral, la sensibilidad aumenta hasta $71,4 \%$ en tumores mayores de $21 \mathrm{~mm}$. La positividad del PET/CT se asoció también al grado de displasia, con sensibilidad de 14,6\% para adenomas de bajo grado, 38,5\% para adenomas de alto grado, 66,7\% para cáncer in situ y $100 \%$ para cáncer invasor. Esta habilidad del PET/CT de detectar más las lesiones de alto grado y el cáncer, le confiere un poder de estratificación de riesgo al enfrentar pacientes con múltiples pólipos, priorizando los recursos en aquellas lesiones que son positivas al PET/CT.

\section{Cáncer sincrónico extracolónico}

La detección de una neoplasia maligna extracolónica sincrónica al CCR ha sido escasamente descrita en la literatura, y existe una gran heterogeneidad respecto a cómo se ha tratado de pesquisar. Por otro lado, varios autores prefieren utilizar el concepto de "neoplasias primarias múltiples", que incluye además los tumores metacrónicos (detectados 6 meses o más, después del CCR), lo que hace aún más difícil conocer la incidencia real de lesiones sincrónicas propiamente tales.

Un estudio coreano encabezado por $\mathrm{Lee}^{13}$ obtiene una frecuencia de $1,47 \%(32 / 2.176)$ de cáncer sincrónico extracolónico detectado hasta 6 meses después del diagnóstico del CCR, con predominancia de cáncer gástrico y tiroideo en su muestra. Ellos no describen los exámenes de imagen o laboratorio utilizados en el estudio inicial ni seguimiento de su población.

En un estudio de cánceres primarios múltiples en población China, Wu et a ${ }^{15}$ obtiene $1,0 \%$ (13/1.311) de cáncer sincrónico extracolónico. Al igual que la casuística coreana, los autores trabajan retrospectivamente con bases de datos poblacionales, y no tienen acceso a la información médica ni exámenes efectuados. En su manuscrito no detallan los métodos diagnósticos empleados, y tampoco separan claramente los tipos de tumores sincrónicos y metacrónicos detectados.

El 5,7\% de cánceres sincrónicos extracolónicos detectados con PET/CTc en nuestra casuística es mayor a lo descrito en los estudios poblacionales mencionados, aunque la metodología empleada en ambas experiencias es bastante distinta a la nuestra. Nosotros utilizamos una población es- tudiada uniformemente con PET/CT y TC contrastada de cuerpo completo, esto es incluyendo cerebro, cuello, tórax abdomen y pelvis, además de un estudio colonoscópico preoperatorio en gran parte de los pacientes. Por otro lado, existió una alta confirmación de los hallazgos incidentales del PET/CTc, aclarándose la causa en $66 \%$ de los incidentalomas. El control clínico riguroso y seguimiento imagenológico en el mismo centro de salud, permite tener acceso a toda la información y evolución a largo plazo, con mayor probabilidad de conocer el significado de los incidentalomas detectados en el PET/CTc inicial.

Una de las limitaciones de nuestro estudio, es el posible sesgo de selección de la población. El 46\% de colonoscopias incompletas de nuestra muestra es mayor a lo reportado en la literatura, lo que sugiere que se trata de una población con mayor frecuencia de tumores obstructivos. Por otro lado, 36\% de frecuencia de metástasis a distancia encontrado, mayor a lo reportado en la literatura $(20-25 \%)^{5-7,22}$, sugeriría que se le realizó PET/ CT de preferencia a aquellos pacientes de mayor riesgo o con sospecha de metástasis. Sin embargo, hay que tener en cuenta la conocida mayor sensibilidad de la técnica híbrida en la detección de metástasis a distancia respecto a la etapificación tradicional ${ }^{19,30,31}$, lo que podría explicar en parte esta diferencia.

\section{Conclusión}

El PET/CTc es capaz de detectar un segundo cáncer primario sincrónico en un porcentaje significativo de pacientes con CCR, con un rol muy destacado del PET especialmente en la pesquisa de lesiones colorrectales, que pueden ser omitidas utilizando sólo la TC contrastada. En casos de colonoscopía incompleta o técnicamente deficiente, el PET/CT resultaría indispensable en el estudio preoperatorio, por cuanto permitiría detectar lesiones proximales en un número significativo de casos. Por otro lado, la TC contrastada es capaz de detectar neoplasias no visibles con PET, como los tumores renales, cánceres de vías urinarias y hepatocarcinomas, y otras lesiones de bajo grado que no presentan avidez por la glucosa marcada. El PET/CTc es una excelente herramienta diagnóstica en CCR, y tendría una especial utilidad en tumores obstructivos infranqueables o en caso de colonoscopías técnicamente deficientes. 


\section{Referencias}

1. GLOBOCAN [Internet]. [citado 16 de Febrero 2019]. Recuperado a partir de: http://globocan.iarc.fr/

2. Cáncer colorrectal en personas de 15 años o más. Guía AUGE. Serie Guías Clínicas, MINSAL, 2013. Disponible en: https://www.minsal.cl/portal/url/item/db8329dc44e6371de0400101640126b5.pdf [Consultado el 16 de febrero de 2019].

3. Arnold M, Sierra MS, Laversanne M, Soerjomataram I, Jemal A, Bray F. Global patterns and trends in colorectal cancer incidence and mortality. Gut 2017; 66: 683-91.

4. Center MM, Jemal A, Smith RA, Ward E. Worldwide Variations in Colorectal Cancer. CA Cancer J Clin 2009; 59: 366-78.

5. van der Geest LG, Lam-Boer J, Koopman M, Verhoef C, Elferink MA, de Wilt JH. Nationwide trends in incidence, treatment and survival of colorectal cancer patients with synchronous metastases. Clin Exp Metastasis 2015; 32: 457-65.

6. van der Pool AE, Damhuis RA, Ijzermans JN, de Wilt $\mathrm{JH}$, Eggermont AM, Kranse R et al. Trends in incidence, treatment and survival of patients with stage IV colorectal cancer: a population-based series. Colorectal Disease 2011; 14: 56-61.

7. Riihimäki M, Hemminki A, Sundquist J, Hemminki K. Patterns of metastasis in colon and rectal cancer. Sci Rep 2016; 6: 29765; doi: 10.1038/srep29765.

8. López-Kostner F, Carrillo K, Zárate A, Obrien A, Ladrón de Guevara D. Cáncer de recto: diagnóstico, estudio y estadificación. Rev Chilena de Cirugía 2012; 64: 199209.

9. Piñol V, Andreu M, Castells A, Payá A, Bessa X, Jover R. Synchronous colorectal neoplasms in patients with colorectal cancer: predisposing individual and familial factors. Dis Colon Rectum 2004; 47: 1192-200.

10. Mulder SA, Kranse R, Damhuis RA, de Wilt JH, Ouwendijk RJ, Kuipers EJ, et al. Prevalence and prognosis of synchronous colorectal cancer: a Dutch population-based study. Cancer Epidemiol 2011; 35: 442-7.

11. Lindberg LJ, Ladelund S, Bernstein I, Therkildsen C, Nilbert M. Risk of synchronous and metachronus colorectal cancer: population- based estimates in Denmark with focus on non-hereditary cases diagnosed after age 50. Scand J Surg 2018; 10: 1-7.

12. Ruffinengo O, Tanno F, Covernton J, Corral M, Curvale C, Robinson J, et al. Colorectal cancer: epidemiologic characteristic in a hospital center of Rosario, Argentina. Acta Gastroenterol Latinam 2010; 40 (2): 98-104.

13. Lee $\mathrm{SH}, \mathrm{Ahn} \mathrm{BK}$, Baek SU. Multiple primary cancers in extracolonic sites with colorectal cáncer. Int J Colorectal
Dis 2009; 24: 301-4.

14. Lee TK, Barringer M, Myers RT, Sterchi JM. Multiple primary carcinomas of the colon and associated extracolonic primary malignant tumors. Ann Surg 1982; 195: 501-7.

15. Wu A, He S, Li J, Liu L, Liu C, Wang Q, et al. Colorectal cancer in cases of multiple primary cancers: Clinical features of 59 cases and point mutation analyses. Oncology Letters 2017; 13: 4720-6.

16. Pickhardt PJ, Kim DH, Meiners RJ, Wyatt KS, Hanson ME, Barlow DS, et al. Colorectal and extracolonic cancers detected at screening CT colonography in 10,286 asymptomatic adults. Radiology 2010; 255: 83-8.

17. National Comprehensive Cancer Network. NCCN Guidelines. Disponible en: http://www.nccn.org/professionals/physician_gls/f_guidelines.asp\#site. [Consultado el 16 de febrero de 2019].

18. Vogel JD, Eskicioglu C, Weiser MR, Feingold DL, Steele SR. The American Society of Colon and Rectal Surgeons Clinical Practice Guidelines for the Treatment of Colon Cancer.

19. Selzner M, Hany TF, Wildbrett P, et al. Does the novel PET/CT imaging modality impact on the treatment of patients with metastatic colorectal cancer of the liver? Ann Surg 2004; 240: 1027-34 [discussion:1035-6].

20. Hall N, Ruutiainen AT. Colorectal Cancer: Imaging Conundrums. Surg Oncol Clin N Am 2017; 27: 289-302.

21. Warren S, Gates O. Multiple primary malignant tumors: a survey of the literature and a statistical study. Am J Cancer 1932; 16: 1358-414.

22. Brenner H, Kloor M, Pox CP. Colorectal cancer. Lancet 2014; 383: 1490-502.

23. Borda A, Martínez -Peñuela JM, Prieto C, Muñoz M, Carretero C, Borda F. Estudio de la frecuencia, distribución y rendimiento diagnóstico en las lesiones neoplásicas sincrónicas del carcinoma colorrectal. An Sist Sanit Navar 2008; 31: 43-52.

24. Shah HA, Paszat LF, Saskin R, Stukel TA, Rabeneck L. Factors associated with incomplete colonoscopy: a populationbased study. Gastroenterology 2007; 132: 2297-303.

25. Mohd Suan MA, Tan WL, Soelar SA, Ismail I, AbuHassan MR. Intestinal obstruction: predictor of poor prognosis in colorectal carcinoma? Epidemiol Health 2015; 37: e2015017. doi: 10.4178/epih/e2015017. eCollection 2015.

26. Hong N, Park SH. CT colonography in the diagnosis and management of colorectal cancer: emphasis on pre-and post-surgical evaluation. World J Gastroenterol 2014; 20: 2014-22.

27. Hojo D, Tanaka T, Takahashi M, Murono K, Emoto S, 
Kaneko M, et al. Efficacy of 18-fluoro deoxy glucose-positron emission tomography computed tomography for the detection of colonic neoplasia proximal to obstructing colorectal cancer. Medicine (Baltimore) 2018; 97 (31): e11655. doi: 10.1097/MD.0000000000011655.

28. Kijima S, Sasaki T, Nagata K, Utano K, Lefor AT, Sugimoto $\mathrm{H}$. Preoperative evaluation of colorectal cancer using CT colonography, MRI, and PET/CT. World J Gastroenterol 2014; 20: 16964-75.

29. Fraum TJ, Ludwing DR, Hope TA, Fowler KJ. PET/MRI for gastrointestinal Imaging Current clinical status and prospects. Gastroenterol Clin North Am 2018; 47: 691714.

30. Kantorová I, Lipská L, Bêlohlávek O, Visokai V, Trubaĉ M, Schneiderová M. Routine (18)F-FDG PET preoperative staging of colorectal cancer: comparison with conventional staging and its impact on treatment decision making. J Nucl Med 2003; 44: 1784-8.

31. Shin SS, Jeong YY, Min JJ, Kim HR, Chung TW, Kang HK. Preoperative staging of colorectal cancer: CT vs. integrated FDG PET/CT. Abdom Imaging 2008; 33: 270-7. 